\title{
AN ELEMENTARY PROOF OF THE STONE-WEIERSTRASS THEOREM
}

\author{
BRUNO BROSOWSKI AND FRANK DEUTSCH ${ }^{1}$
}

ABSTRACT. An elementary proof of the Stone-Weierstrass theorem is given.

In this note we give an elementary proof of the Stone-Weierstrass theorem. The proof depends only on the definitions of compactness ("each open cover has a finite subcover") and continuity ("the inverse images of open sets are open"), two simple consequences of these definitions (i.e. "a closed subset of a compact space is compact," and "a positive continuous function on a compact set has a positive infimum"), and the elementary Bernoulli inequality:

if $h>-1$.

$$
(1+h)^{n} \geqslant 1+n h \quad(n=1,2, \ldots)
$$

In the beautiful and elementary proof of the classical Weierstrass theorem given by Kuhn [1], it is observed that it suffices to be able to approximate, by polynomials, the step function which is 1 on the interval $\left[0, \frac{1}{2}\right)$ and 0 on the interval $\left[\frac{1}{2}, 1\right]$ uniformly outside of each neighborhood of $\frac{1}{2}$. The main step in our proof (Lemma 2 ) is the general analogue of this. It shows that it suffices to be able to approximate, by elements of the subalgebra, a given "generalized step function" (i.e. a function which is $\mathbf{0}$ on a closed set and $\mathbf{1}$ off the set) uniformly on the closed set and off a neighborhood of this set.

It should be remarked that when our proof is specialized to the classical case of polynomials in $C[a, b]$, it is even "simpler" than Kuhn's proof in the sense that no "change of variables" argument is necessary, nor is it necessary to appeal to the fact that continuous functions on $[a, b]$ are uniformly continuous. (Kuhn's proof also used the Bernoulli inequality.)

In particular, it is perhaps worth emphasizing that, in contrast to many proofs of the Stone-Weierstrass theorem, we do not appeal to any of the following facts:

(a) the classical Weierstrass theorem (nor even the special case of uniformly approximating $f(t)=|t|$ on $[-1,1]$ by polynomials);

(b) that the closure of a subalgebra is a subalgebra;

(c) that the closure of a subalgebra is a sublattice.

Let $T$ be a compact topological space and $C(T)$ the set of all real-valued continuous functions on $T$. A neighborhood of a point in $T$ is an open set which

Received by the editors July 10, 1979 and, in revised form, April 24, 1980.

1980 Mathematics Subject Classification. Primary 46J30, 46E15; Secondary 41 A30.

Key words and phrases. Stone-Weierstrass theorem, Weierstrass theorem, approximation by subalgebras, polynomial approximation.

'On leave from the Pennsylvania State University, University Park, Pennsylvania 16802. 
contains the point. Let $\mathfrak{A}$ be a subset of $C(T)$ with the properties:

(i) $x, y$ in $\mathfrak{A}, \alpha, \beta$ in $\mathbf{R}$ implies $\alpha x+\beta y \in \mathfrak{A}$;

(ii) $x, y$ in $\mathfrak{A}$ implies $x \cdot y \in \mathfrak{U}$;

(iii) $1 \in \mathfrak{A}$;

(iv) if $t_{1}$ and $t_{2}$ are distinct points in $T$, then there exists $x \in \mathfrak{A}$ such that $x\left(t_{1}\right) \neq x\left(t_{2}\right)$.

In other words, $\mathfrak{A}$ is a "subalgebra of $C(T)$ which contains constants and separates points."

The Stone-Weierstrass theorem may be stated as follows. If $\mathfrak{A}$ is a subalgebra of $C(T)$ which contains constants and separates points, then the elements of $C(T)$ can be uniformly approximated by the elements of $\mathfrak{A}$. That is, given $f \in C(T)$ and $\varepsilon>0$ there exists $g \in \mathfrak{A}$ such that $\sup _{t \in T}|f(t)-g(t)|<\varepsilon$.

It is convenient to divide the proof into three steps. The essential step is Lemma 1. For brevity, the norm notation $\|x\|=\sup \{|x(t)| \mid t \in T\}$ will sometimes be used.

Lemma 1. Let $t_{0} \in T$ and let $U$ be $a$ neighborhood of $t_{0}$. Then there is $a$ neighborhood $V=V\left(t_{0}\right)$ of $t_{0}, V \subset U$, with the following property. For each $\varepsilon>0$, there exists $x \in \mathfrak{A}$ such that

(1) $0<x(t)<1, t \in T$

(2) $x(t)<\varepsilon, t \in V$;

(3) $x(t)>1-\varepsilon, t \in T \backslash U$.

Proof. For each $t \in T \backslash U$, the point separating property (iv) implies that there is a function $g_{t} \in \mathfrak{A}$ with $g_{t}(t) \neq g_{t}\left(t_{0}\right)$. Then the function $h_{t}=g_{t}-g_{t}\left(t_{0}\right) \cdot 1$ is in $\mathfrak{A}$ and $h_{t}(t) \neq h_{t}\left(t_{0}\right)=0$. The function $p_{t}=\left(1 /\left\|h_{t}^{2}\right\|\right) h_{t}^{2}$ is in $\mathfrak{A}, p_{t}\left(t_{0}\right)=0, p_{t}(t)>0$, and $0<p_{t}<1$.

Let $U(t)=\left\{s \in T \mid p_{t}(s)>0\right\}$. Then $U(t)$ is a neighborhood of $t$. By compactness of $T \backslash U$, there exist a finite number of points $\left\{t_{1}, t_{2}, \ldots, t_{m}\right\}$ in $T \backslash U$ such that $T \backslash U \subset \cup{ }_{1}^{m} U\left(t_{i}\right)$. Let $p=(1 / m) \sum_{1}^{m} p_{t_{i}}$. Then $p \in \mathfrak{A}, 0<p<1, p\left(t_{0}\right)=0$, and $p>0$ on $T \backslash U$.

Again using the compactness of $T \backslash U$, there exists $0<\delta<1$ such that $p>\delta$ on $T \backslash U$. Let $V=\{t \in T \mid p(t)<\delta / 2\}$. Then $V$ is a neighborhood of $t_{0}$ and $V \subset U$.

Let $k$ be the smallest integer which is greater than $1 / \delta$. Then $k-1<1 / \delta$ which implies that $k \leqslant(1+\delta) / \delta<2 / \delta$. Thus $1<k \delta<2$. Consider the functions $q_{n}$ defined by

$$
q_{n}(t)=\left[1-p^{n}(t)\right]^{k^{n}} \quad(n=1,2, \ldots)
$$

Clearly, $q_{n} \in \mathfrak{A}, 0 \leqslant q_{n} \leqslant 1$, and $q_{n}\left(t_{0}\right)=1$. For each $t \in V, k p(t)<k \delta / 2<1$ so that, by Bernoulli's inequality,

$$
q_{n}(t) \geqslant 1-[k p(t)]^{n} \geqslant 1-(k \delta / 2)^{n} \rightarrow 1
$$

uniformly on $V$. For $t \in T \backslash U, k p(t)>k \delta>1$ and, using Bernoulli's inequality, 


$$
\begin{aligned}
q_{n}(t) & =\frac{1}{k^{n} p^{n}(t)}\left[1-p^{n}(t)\right]^{k^{n}} k^{n} p^{n}(t)<\frac{1}{[k p(t)]^{n}}\left[1-p^{n}(t)\right]^{k^{n}}\left[1+k^{n} p^{n}(t)\right] \\
& <\frac{1}{[k p(t)]^{n}}\left[1-p^{n}(t)\right]^{k^{n}}\left[1+p^{n}(t)\right]^{k^{n}}=\frac{1}{[k p(t)]^{n}}\left[1-p^{2 n}(t)\right]^{k^{n}} \\
& <\frac{1}{(k \delta)^{n}} \rightarrow 0
\end{aligned}
$$

uniformly on $T \backslash U$.

Thus for $n$ sufficiently large, the function $q_{n}$ has the property that $0<q_{n}<1$, $q_{n}<\varepsilon$ on $T \backslash U$, and $q_{n}>1-\varepsilon$ on $V$. The result follows by taking $x=1-q_{n}$.

LEMMA 2. Let $A$ and $B$ be disjoint closed subsets of $T$. Then for each $0<\varepsilon<1$, there exists $x \in \mathfrak{A}$ such that

(1) $0<x(t) \leqslant 1, t \in T$;

(2) $x(t)<\varepsilon, t \in A$;

(3) $x(t)>1-\varepsilon, t \in B$.

Proof. Let $U=T \backslash B$. For each $t \in A$, choose the neighborhood $V(t)$ of $t$ as in Lemma 1. Then there exists a finite set of points $\left\{t_{1}, t_{2}, \ldots, t_{m}\right\}$ in $A$ such that $A \subset \cup{ }_{1}^{m} V\left(t_{i}\right)$. By the choice of $V\left(t_{i}\right)$, there exist $x_{i} \in \mathfrak{A}(i=1,2, \ldots, m)$ with $0 \leqslant x_{i} \leqslant 1, x_{i}<\varepsilon / m$ on $V\left(t_{i}\right)$, and $x_{i}>1-\varepsilon / m$ on $T \backslash U=B$. Then the function $x=x_{1} \cdot x_{2} \cdots x_{m}$ is in $\mathfrak{A}, 0<x<1, x<\varepsilon / m<\varepsilon$ on $\cup_{1}^{m} V\left(t_{i}\right) \supset A$, and (using Bernoulli's inequality) $x>(1-\varepsilon / m)^{m}>1-\varepsilon$ on $B$.

Finally, we turn to the proof of the Stone-Weierstrass theorem. Let $f \in C(T)$ and $\varepsilon>0$. To complete the proof, it suffices to show the existence of a $g \in \mathfrak{A}$ such that

$$
|f(t)-g(t)|<2 \varepsilon, \quad t \in T .
$$

By replacing $f$ by $f+\|f\|$, we can assume that $f \geq 0$. We may also assume that $\varepsilon<\frac{1}{3}$. Choose an integer $n$ so that $(n-1) \varepsilon>\|f\|$. Define the sets $A_{j}, B_{j}(j=$ $0,1, \ldots, n)$ by

$$
A_{j}=\left\{t \in T \mid f(t) \leqslant\left(j-\frac{1}{3}\right) \varepsilon\right\}, \quad B_{j}=\left\{t \in T \mid f(t)>\left(j+\frac{1}{3}\right) \varepsilon\right\} .
$$

Note that $A_{j}$ and $B_{j}$ are disjoint closed sets in $T, \varnothing=A_{0} \subset A_{1} \subset \cdots \subset A_{n}=T$, and $B_{0} \supset B_{1} \supset \cdots \supset B_{n}=\varnothing$. For each $j=0,1, \ldots, n$, Lemma 2 implies that there is $x_{j} \in \mathfrak{A}$, with $0 \leqslant x_{j} \leqslant 1, x_{j}<\varepsilon / n$ on $A_{j}$, and $x_{j}>1-\varepsilon / n$ on $B_{j}$.

Then the function $g=\varepsilon \sum_{0}^{n} x_{i}$ is in $\mathfrak{A}$. For any $t \in T$, we have $t \in A_{j} \backslash A_{j-1}$ for some $j \geqslant 1$ which implies that

$$
\left(j-\frac{4}{3}\right) \varepsilon<f(t) \leqslant\left(j-\frac{1}{3}\right) \varepsilon
$$

and

$$
x_{i}(t)<\varepsilon / n \text { for every } i \geqslant j .
$$

Also, $t \in B_{i}$ for every $i \leqslant j-2$ which implies

$$
x_{i}(t)>1-\varepsilon / n \text { for every } i<j-2 .
$$


Using (***), we obtain

$$
\begin{aligned}
g(t) & =\varepsilon \sum_{0}^{j-1} x_{i}(t)+\varepsilon \sum_{j}^{n} x_{i}(t) \\
& \leqslant j \varepsilon+\varepsilon(n-j+1) \varepsilon / n<j \varepsilon+\varepsilon^{2}<\left(j+\frac{1}{3}\right) \varepsilon .
\end{aligned}
$$

Using (****), we obtain for $j \geqslant 2$

$$
\begin{aligned}
g(t) & \geqslant \varepsilon \sum_{0}^{j-2} x_{i}(t) \geqslant(j-1) \varepsilon(1-\varepsilon / n) \\
& =(j-1) \varepsilon-((j-1) / n) \varepsilon^{2}>(j-1) \varepsilon-\varepsilon^{2}>\left(j-\frac{4}{3}\right) \varepsilon .
\end{aligned}
$$

The inequality $g(t)>\left(j-\frac{4}{3}\right) \varepsilon$ is trivially true for $j=1$. Thus

$$
|f(t)-g(t)| \leqslant\left(j+\frac{1}{3}\right) \varepsilon-\left(j-\frac{4}{3}\right) \varepsilon<2 \varepsilon .
$$

\section{REFERENCES}

1. H. Kuhn, Ein elementarer Beweis des Weierstrasschen Approximationssatzes, Arch. der Math. (Basel) 15 (1964), 316-317.

Fachbereich Mathematik, J. W. Goethe-Universität Frankfurt, Robert Mayer-Strasse 6-10, D-6000 Frankfurt, West Germany (Current address of Bruno Brosowski)

Current address (Frank Deutsch): Department of Mathematics, Pennsylvania State University, University Park, Pennsylvania 16802 